\title{
Blind Super-Resolution Approach for Exploiting Illumination Variety in Optical-Lattice Illumination Microscopy
}

\author{
Krishnendu Samanta, Swagato Sarkar, Sebastian Acuña, Joby Joseph,* Balpreet Singh Ahluwalia, \\ and Krishna Agarwal*
}

Cite This: ACS Photonics 2021, 8, 2626-2634

Read Online

\section{ACCESS \\ Џlll Metrics \& More \\ Article Recommendations \\ Supporting Information}

ABSTRACT: Optical-lattice illumination patterns help in pushing high spatial frequency components of the sample into the optical transfer function of a collection microscope. However, exploiting these high-frequency components require precise knowledge of illumination if reconstruction approaches similar to structured illumination microscopy are employed. Here, we present an alternate blind reconstruction approach that can provide superresolution without the requirement of extra frames. For this, the property of exploiting temporal fluctuations in the sample emissions using "multiple signal classification algorithm" is
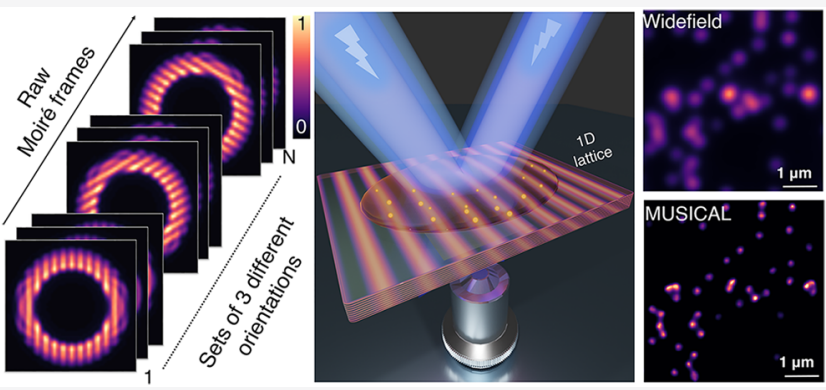
extended aptly toward using spatial fluctuation of phase-modulated lattice illuminations for super-resolution. The super-resolution ability is shown for sinusoidal and multiperiodic lattice with approximately 3- and 6-fold resolution enhancements, respectively, over the diffraction limit.

KEYWORDS: fluorescent imaging, optical microscopy, lattice illumination, eigen-image analysis, blind reconstruction, super-resolution

$\mathrm{O}$ ver the past few years, a variety of optical superresolution techniques have been launched to improve the diffraction limit of resolution ${ }^{1,2}$ toward nanoscale dimensions, eventually leading to a new branch of optics called "optical nanoscopy". 3,4 One of the prevalent approaches of the super-resolution techniques is based on single-molecule detection where a random set of molecules are excited at different times via photoswitching, which includes photoactivated localization microscopy (PALM), ${ }^{5,6}$ stochastic optical reconstruction microscopy (STORM), ${ }^{7,8}$ and so on. This has a major drawback, as it requires a large number of frames $(\sim 1000 s)$, which slows down the process of imaging. Another broad category uses modulation of diffraction-limited excitation spot with a depletion pulse to sharpen the excitation point-spread-function optically, stimulated emission depletion (STED) $)^{9,10}$ and ground-state depletion (GSD) ${ }^{11,12}$ belong to this category. These techniques are complicated and severely phototoxic for the biological samples. As an alternative efficient and robust technique, structured illumination microscopy $(\mathrm{SIM})^{13,14}$ utilizes a sinusoidal illumination pattern and provides 2 -fold resolution enhancement than the diffraction limit. Despite offering lesser resolution compared to STED and STORM, SIM is advantageous in terms of temporal resolution, experimental simplicity, and compatibility with live-cell imaging. In a large-scale scenario, structured illumination can be extended beyond the sinusoidal illumination, generalized as optical lattice illumination. Optical lattices are the spatially varying intensity distributions with periodic arrangements embedded in a 3D volumetric region. These lattices possess potential applications in different fields of science and technology including transport and trapping of metal nanoparticles, ${ }^{15}$ trapping of ultracold atoms by periodic potential, ${ }^{16}$ quantum computation, ${ }^{17}$ and so on. Excitation strategies for complex optical lattices have been proposed theoretically, ${ }^{18}$ although experimental implementation of such lattices may be somewhat challenging.

In this Article, we envisage the utility of optical lattice illumination and propose a super-resolution imaging scheme without assuming the knowledge of the illumination patterns. Sinusoidal and multiperiodic patterns are considered with three different orientations (for isotropy) that are generated by phase engineered interference of coplanar beams. In both cases, the raw Moire frames are acquired using a test object with multiple "phase-shifted" lattice illuminations. The phaseshifting is executed in consecutive steps such that the average of all the recorded frames over the entire acquisition remains uniform. Fourier domain reconstruction algorithms ${ }^{19,20}$ require precise knowledge of the illumination spatial frequency and phase corresponding to each image. A slight error in this

Received: April 2, 2021

Published: August 19, 2021 


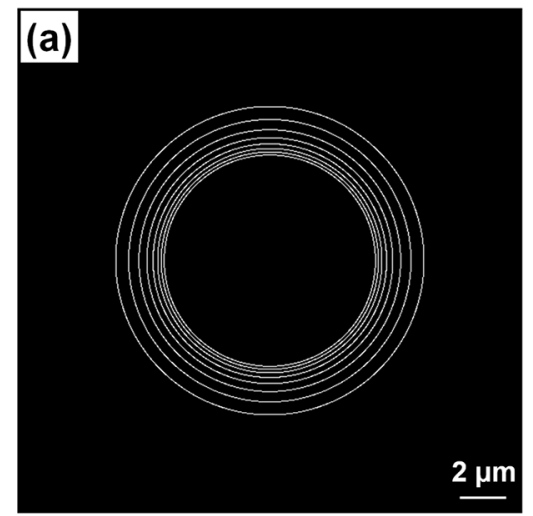

(f)

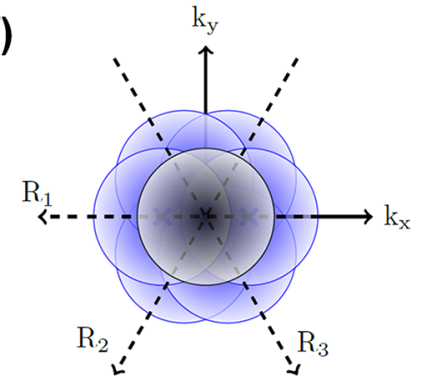

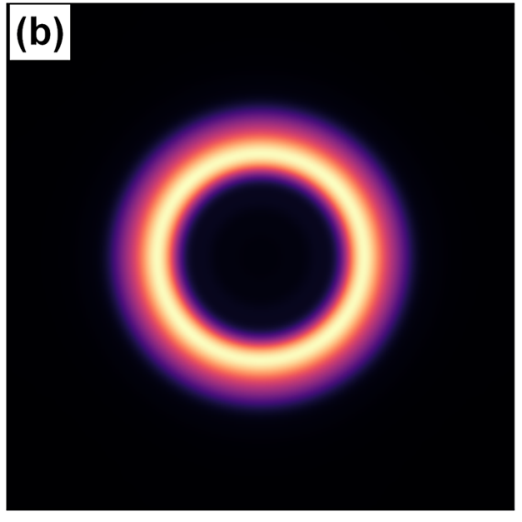

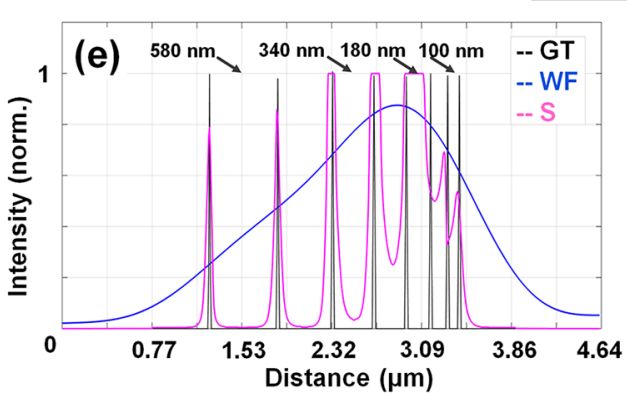

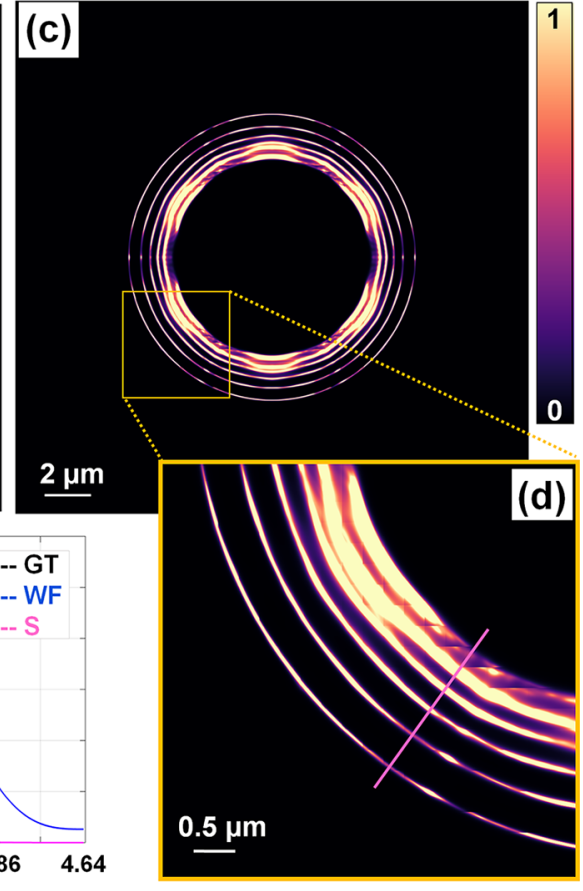

Figure 1. Example structure and simulation results of acquired data using sinusoidal lattice illumination and processing with MUSICAL: (a) ground-truth sample; (b) widefield image for the emission $\left(\lambda_{\text {em }}\right)=450 \mathrm{~nm}$ and NA $=0.3$; $(c)$ reconstructed image obtained using MUSICAL; $(d)$ magnified view of a small portion of (c); (e) radial line-scan of ground-truth, widefield, and reconstructed image; (f) spatial frequency spectra of the imaging configuration for the lattice illumination in three different orientations $R_{1}, R_{2}$, and $R_{3}$.

information causes significant artifacts in the final reconstructed image, ${ }^{21,22}$ and elaborate estimation approaches may be needed as an overhead, only marginally improving the reconstruction. Since the knowledge of illumination patterns is not considered, a blind reconstruction approach is required in our case. For this, we consider that the change in illumination patterns results in changes in fluorescence emission at a given point in the sample over time. Therefore, methods that use temporal variations in fluorescence intensity are the candidates for reconstruction algorithms. However, most of these methods are based on the assumption that each fluorescent emitter behaves independent to other emitters in terms of photoemissions. This condition is violated in the case of optical-lattice illuminations since photoemissions from different emitters are a function of the spatial distribution of illumination patterns and are, therefore, not independent. Thus, we use multiple signal classification algorithm (MUSICAL), ${ }^{23}$ which does not make this assumption and can also use unknown systematic variations in fluctuation intensities over space and time. This has been shown for moving vesicles, ${ }^{24}$ where unknown systematic variation in fluorescence intensity arises from the motion of vesicles. The raw frames over the entire acquisition in such cases are processed MUSICAL-ly to generate a super-resolved image, where MUSICAL exploits the systematic variation in illumination distribution for super-resolution.

In this work, MUSICAL creates a bridge that connects temporal fluctuation and spatial fluctuation based imaging, together. The use of a periodic pattern in MUSICAL requires only as many raw images as needed for diffraction-limited isotropic optical-lattice microscopy images, which is significantly lesser than the number of frames conventionally used in MUSICAL ( 100s). This implies super-resolution at no extra trade-off in temporal resolution, having a direct advantage against a limited photon budget. It also implies that a special arrangement for speckle-like or pseudorandom illumination can be excluded by the current approach of performing SIM without the knowledge of the illumination patterns. ${ }^{25-28}$ Further, as compared to the recent demonstrations of applying fluctuation-based technique, ${ }^{29}$ SOFI coupled with SIM where $\sim 100$ s of frames per illumination pattern are used, we use only one frame per illumination pattern. The efficiency of MUSICAL as a lattice illumination microscopy reconstruction is explained through the eigen-image analysis.

\section{RESULTS AND DISCUSSION}

The optical lattice illumination schemes with periodicity along a particular direction are explored for the evaluation of the MUSICAL performance. This lattice can be strategically generated in a reconfigurable optical setup by the phaseengineered interference $^{30}$ of multiple coplanar beams that allows easy manipulation of spatial fluctuations. Implementation of the periodic pattern is advantageous, as a uniform average can be attained using a lesser number of frames compared to any other illumination schemes. For example, there is a requirement of a large number of frames $(>1000)$ for fluctuation-based imaging techniques such as SOFI ${ }^{31}$ when uniform illumination is used; conversely, in randomly distributed speckle illumination-based, super-resolution imaging, $^{26}$ a similar criterion is a prerequisite. For the current illumination strategies with 1D lattices, isotropy can be achieved by considering different planes of incidence of the interfering beams along different orientations that has been discussed with reference to the following cases.

MUSICAL in Sinusoidal Lattice Illumination. Sinusoidal (single-modal) optical lattice illumination microscopic imaging 

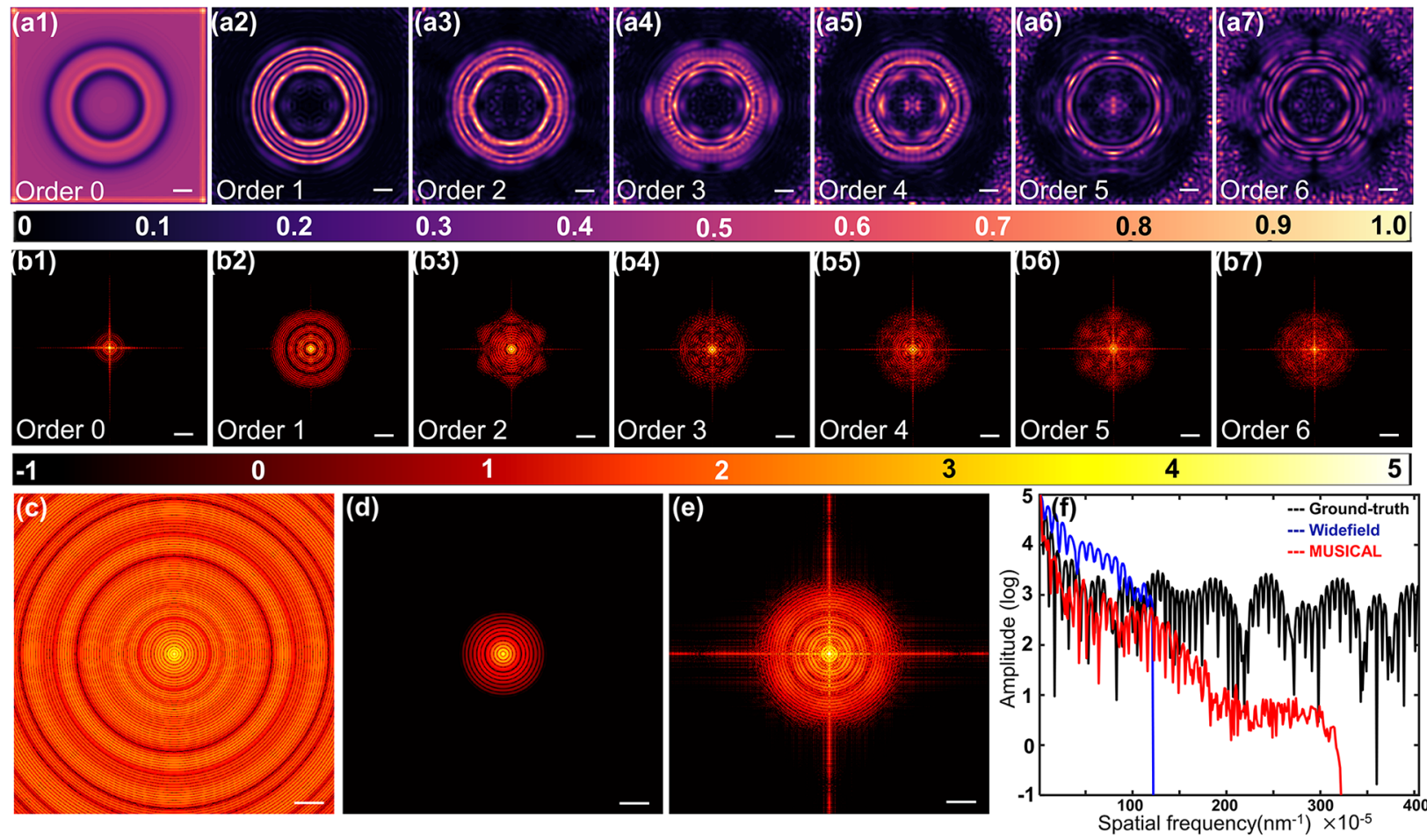

Figure 2. (a1-a7) Projection of different orders or eigen-images displayed in increasing order, scale bar $=2 \mu \mathrm{m}$; (b1-b7) Fourier spectra of the eigenimages (a1)-(a7) displayed in the $\log _{10}$ scale, scale bar $=0.8 \mu \mathrm{m}^{-1}$; (c-e) spectra of the ground-truth sample, widefield image, and MUSICAL image; (f) plot of the normalized power spectral spread.

scheme is rather straightforward, where two beams interfere in the sample region at an angle $\theta$; the half interference angle between the interfering beams. The spatial periodicity is along a particular orientation in the $x-y$ plane depending on the plane of incidence, and there is no axial periodicity of the lattice. Three different orientations, $R_{1}, R_{2}$, and $R_{3}$, are considered to incorporate isotropic lattice illumination to the sample. The spatial periodicity of the lattice illumination pattern is $\frac{\lambda_{\mathrm{ex}}}{2 \sin \theta}$, where $\lambda_{\mathrm{ex}}$ is the excitation wavelength of the fluorescence dye used for labeling the sample. In the simulation, half of the interference angle is taken as $\theta=10^{\circ}$; the excitation wavelength $\left(\lambda_{\mathrm{ex}}\right)$ and emission wavelength $\left(\lambda_{\mathrm{em}}\right)$ of the fluorescent dye are chosen as 400 and $450 \mathrm{~nm}$, respectively. The resultant irradiance profile of the two-beam interference in $R_{j}$ orientation is represented by the following equation:

$$
I_{j, \delta \psi}(\boldsymbol{r})=\left|\boldsymbol{E}_{-1}^{R_{j}}\right|^{2}+\left|\boldsymbol{E}_{+1}^{R_{j}}\right|^{2}+2\left|\boldsymbol{E}_{-1}^{R_{j}}\right|\left|\boldsymbol{E}_{+1}^{R_{j}}\right| \cos \left(\delta \boldsymbol{k}^{R_{j} \cdot r}+\delta \psi\right)
$$

where $\left|\delta \boldsymbol{k}^{R_{j}}\right|=2 k \sin \theta$ is the difference between the wave vectors, $\delta \psi$ is the phase difference of the interfering beams, and $\boldsymbol{E}$ denotes the electric field vector of the interfering beams. Let the sample be assumed to be two-dimensional and the density function of the fluorophore within the sample to be $S(r)$. The ground-truth synthetic object chosen for the simulation representing the fluorescent sample is concentric circular rings with varying separations of 580, 450, 340, 250, 180, 130, and $100 \mathrm{~nm}$ from outside toward the center, respectively, which is shown in Figure 1a. The sample is illuminated by the series of sinusoidal lattice patterns, and a set of images have been acquired using a standard fluorescent microscopy system that comprises an objective lens $(\mathrm{NA}=0.3)$, a matched tube lens, emission wavelength filter, and imaging sensor or camera (square pixel of size $6.5 \mu \mathrm{m}$ ). The Moire pattern for lattice illumination with phase difference $\delta \psi$ in $R_{j}$ orientation is expressed as

$$
D_{j, \delta \psi}(\boldsymbol{r})=\left[S(\mathbf{r}) I_{j, \delta \psi}(\boldsymbol{r})\right] \otimes h(\boldsymbol{r})
$$

where $h(r)$ is the point spread function of the imaging system and $\otimes$ is the convolution operator. Figure 1f represents the spatial frequency spectra $\left(k_{x}-k_{y}\right)$ of the imaging system with lattice illumination where near-isotropic coverage of spectraldomain is shown in three different orientations $\left(R_{1} ; R_{2} ; R_{3}\right)$. In each of these three orientations, lattice illumination $I_{j, \delta y}(\boldsymbol{r})$ undergoes phase-shifting in small steps along the direction of its periodicity in such a way that the average over all steps leads to a uniform illumination for that orientation. Uniform average is the main condition for an appropriate reconstruction; otherwise, information from some portion of the sample will be missing in the final image. Here a $0.2 \pi$ phase step is used for simulation, and a total of 30 images (10 in each orientation) are recorded. The simulation consists of two steps: In the first step, the raw microscopy data or Moire frames are generated with different orientations and phase steps. By simulating the raw time traces in a scheme discussed above, each Moiré pattern contains information from different domains of fluorophores depending on the lattice illumination. In the second step, the information contained within the raw data over the entire acquisition process has been utilized in a computational scheme to reconstruct a final image. The ImageJ plugin $^{32}$ for MUSICAL has been used, and the parameters used for the MUSICAL plugin are the threshold singular value $\left(\lambda_{\text {th }}\right)=-3$ and the number of subpixels per pixel $=10$. Figure $\mathrm{lb}$ is the widefield image of the sample; Figure $1 \mathrm{c}$ is the MUSICAL reconstructed image; and Figure $1 \mathrm{~d}$ is the 


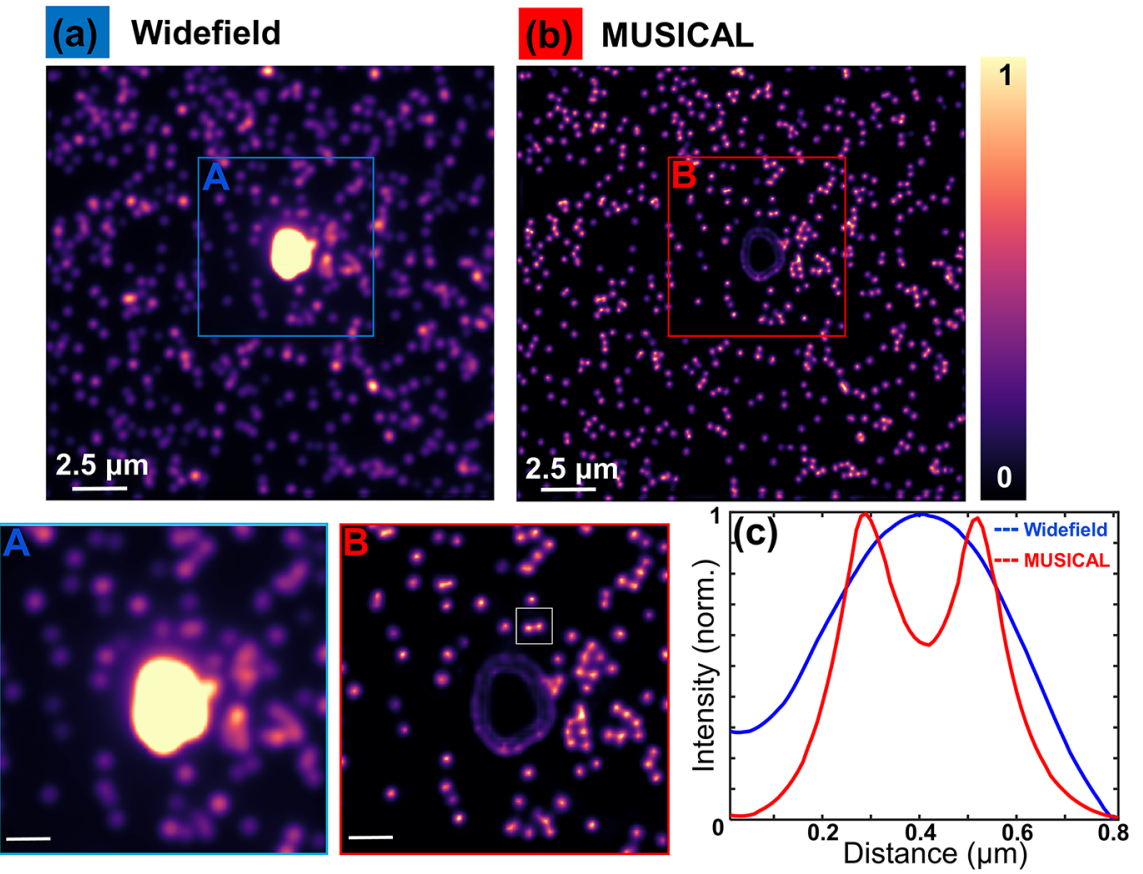

Figure 3. Experimental results with $100 \mathrm{~nm}$ (diameter) fluorescent beads sample: (a) widefield image; (b) MUSICAL reconstruction; (A, B) the magnified version of a selected region of (a) and (b) with a scale bar $=1 \mu \mathrm{m}$; (c) intensity scan profile across a line within the selected box.

magnified view of a small portion of the reconstructed image. It is seen that there are artifacts present along three orientations $R_{1}, R_{2}$, and $R_{3}$; it is a known fact that illumination contributes artifacts in the reconstructed image of structured illumination microscopy ${ }^{19,33}$ and chip-based nanoscopy with nonuniform illumination. ${ }^{28}$ The presence of such angle-specific artifacts indicates that this is primarily caused by the illumination rather than the reconstruction. However, the postprocessing algorithms can be designed to compensate for such artifacts. ${ }^{34}$ Figure 1e shows the radial line-scan of ground-truth, widefield, and final super-resolved image of the fluorescent sample. From the radial line scan profile, it is observed that separations more than $250 \mathrm{~nm}$ are fairly resolved, which signifies that we achieve around 3-fold lateral resolution enhancement over the diffraction limit $(750 \mathrm{~nm})$ using the sinusoidal lattice illumination.

MUSICAL classifies the spatiotemporal information contained within the raw image sequence applying the singular value decomposition. A brief summary of the MUSICAL algorithm employed in the lattice illumination scheme is shown in the Supporting Information, T1. A user-defined threshold divides the set of eigenimages into two sections: signal and noise. Only a certain number of eigenimages play a significant role in achieving super-resolution; these belong to the signal space. Other eigenimages from the noise space have no contribution to the resolution. Figure $2 \mathrm{a} 1-\mathrm{a} 7$ shows the projections of the first seven eigenimages, and Figure $2 b 1-b 7$ represents the corresponding Fourier spectra. The complete set of eigenimages and eigenspectra are shown in the Supporting Information, T2. It is seen from Figure 2a1 that there is hardly any resolution enhancement; this is because the first eigenimage (0th order) corresponds to the mean image, which is equivalent to the widefield image. This is also affirmed through the small spread in the frequency domain shown in Figure $2 \mathrm{~b} 1$. Super-resolution information is present from first order onward, as evident in Figure 2a2-a7 and, correspond- ingly, Figure $2 \mathrm{~b} 2-\mathrm{b} 7$ in the frequency domain. We also see that each image in Figure 2a2-a7 contains condensed information from all the probing frequencies symmetrically. This is because the eigen-decomposition takes all the frames as constructs condensed information in each eigenimage. However, owing to the orthogonality of the eigenimages, each image in Figure 2a2-a7 contains complementary information about the sample, as more strongly evident in the frequency spectra in Figure $2 \mathrm{~b} 2-\mathrm{b} 7$. The illumination patterns contribute to the signal space and are orthogonal to the noise space. Since there are six frequency nodes and a widefield component in the sinusoidal lattice (for three orientations), the signal space comprises only seven eigenimages, and the rest are attributed to the noise space. The denominator in contrast uses the orthogonality of eigenimages in the noise space with the illumination patterns, therefore, indirectly and nonlinearly exploiting the illumination patterns further. Figure $2 c-f$ corresponds to the spectra of the ground-truth sample, widefield image, the MUSICAL reconstructed image, and the normalized power spectra analysis, respectively. The net MUSICAL spectrum shown in Figure 2e, therefore, covers a significantly larger region as compared to the original diffraction limited span shown in Figure 2d.

To verify the resolution enhancement obtained using the proposed approach, we performed an experiment with fluorescent beads (100 nm diameter, T7279 Tetraspeck, Thermofisher) imaged using an optical lattice, and processed using MUSICAL. The details of the experimental methods are provided in Supporting Information, T5. A set of nine raw Moire frames (three frames with three orientations) are recorded by the microscope with imaging objective lens $\mathrm{NA}=$ 1.2; excitation and emission wavelengths are 660 and $690 \mathrm{~nm}$, respectively. Figure $3 \mathrm{a}$ and $\mathrm{b}$ represent the widefield (diffraction-limited) and MUSICAL images; Figure 3A and B correspond to the magnified version of a region of (a) and (b), 

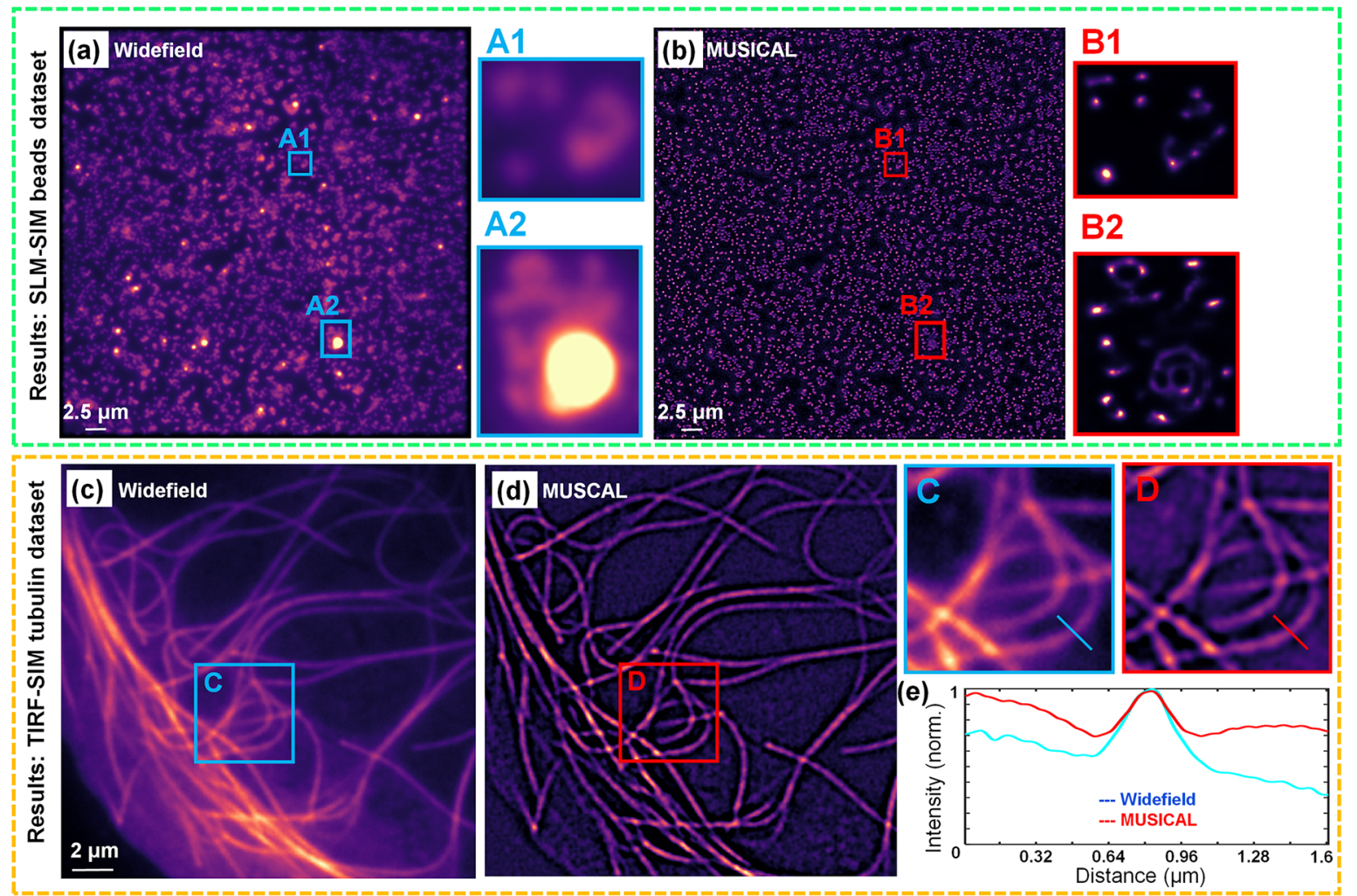

Figure 4. (a, b) MUSICAL analysis on SLM-SIM fluorescent bead data set: (a) widefield image and (b) MUSICAL reconstruction. B1 and B2 corresponds to the MUSICAL-ly reconstructed versions of the magnified sections A1 and A2 in the widefield image. (c-e) Similar analysis on TIRF-SIM tubulin data set: (c) widefield image and (d) MUSICAL reconstruction. "D" corresponds to the MUSICAL-ly reconstructed version of the section "C" in the widefield image (e) intensity scan profile along a line segment shown in images C and D.

respectively; Figure $3 \mathrm{c}$ is the intensity profile across a line. Two fluorescent beads with a separation of $246 \mathrm{~nm}$ are nicely resolved in the case of MUSICAL; which are unresolved in the widefield image. There is an interesting region in the sample containing a high density of fluorophores where the beadcluster gives rise to the camera-saturation level. The intensity variation incorporated by the optical lattice within this region becomes insignificant for the reconstruction. Therefore, MUSICAL cannot provide true reconstruction for this kind of region. We note that MUSICAL, similar to most nonlinear statistical reconstruction algorithms, faces challenges in the case of nonsparse objects. ${ }^{38}$ In such an event, linearized versions of MUSICAL ${ }^{38}$ can be suitable alternatives. The performance of MUSICAL over standard algorithms for SIM is shown through a comparative analysis in Figure S4 of the Supporting Information, T3.

To check the quality of the reconstructed image, we have implemented MUSICAL on a few more samples. Freely available raw SIM test data sets found from the open access repository ${ }^{19}$ have been utilized. Raw data of fluorescent beads sample are tested where a total of 12 frames (four orientations and three phase steps per orientation) are processed with an emission wavelength is $680 \mathrm{~nm}$ and $\mathrm{NA}=1.2$. The widefield image is shown in Figure 4a, where (A1) and (A2) are two selected magnified regions. The MUSICAL image is shown in Figure $4 \mathrm{~b}$, where (B1) and (B2) are same magnified regions. This affirms the fact that MUSICAL can be robustly utilized as a standard reconstruction approach for raw data of lattice illumination microscopy obtained from any commercial instruments or custom-built systems. Another raw data set of the tubulin sample recorded using TIRF-SIM setup is checked, where a total of nine frames (three orientations and three phase steps per orientation) are processed with MUSICAL with emission wavelength is $525 \mathrm{~nm}$ and $\mathrm{NA}=1.49$. This is a typical case where illumination frequency lies outside the optical passband limit. The results shown in the bottom row of Figure $4 c, d$ correspond to the widefield and MUSICAL images, respectively, Figure $4 \mathrm{e}$ is the intensity scan profile along a line segment. The results from the tubulin data show that there is hardly any difference between the widefield and MUSICAL image; MUSICAL fails to provide any resolution improvement for this data. We consider this example in more detail in Supporting Information, T6 and Figure S7. In the situation where the illumination patterns can indeed contribute fluctuations, MUSICAL does provide super-resolution. In this sense, while analyzing the suitability of illumination patterns for MUSICAL, it is useful to assess both the average illumination and the standard deviation of the illumination.

MUSICAL in Multiperiodic Lattice Illumination. The wider the range of spatial frequencies collected by the imaging system, the higher is the achievable resolution, which reflects the fact that high-frequency lattice illumination would lead to a better resolution. However, from the analysis of the sinusoidal lattice, we notice that MUSICAL fails to provide any resolution improvement for lattice illumination when the illumination frequency exists outside the optical passband. This section deals with an approach to bypass the limitation of MUSICAL and to provide further resolution enhancement. It can be achieved through a transmission configuration where the illumination path is decoupled from the collection path. In transmission geometry, we describe a scheme where the sample is illuminated with a multiperiodic one-dimensional 

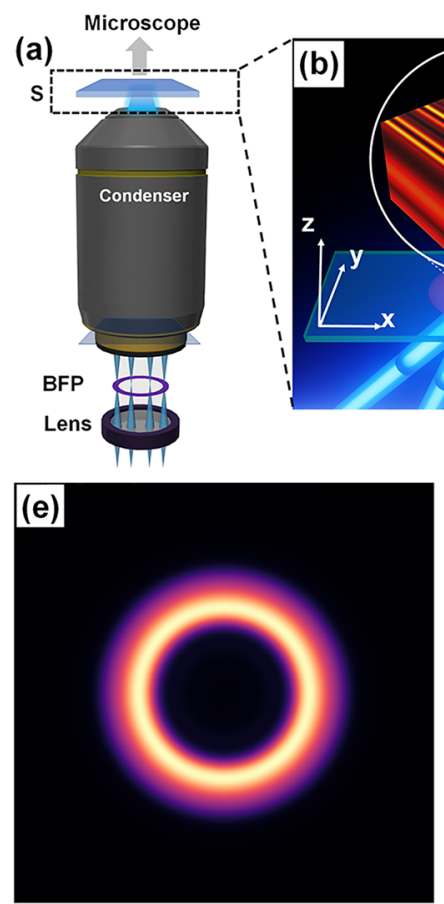
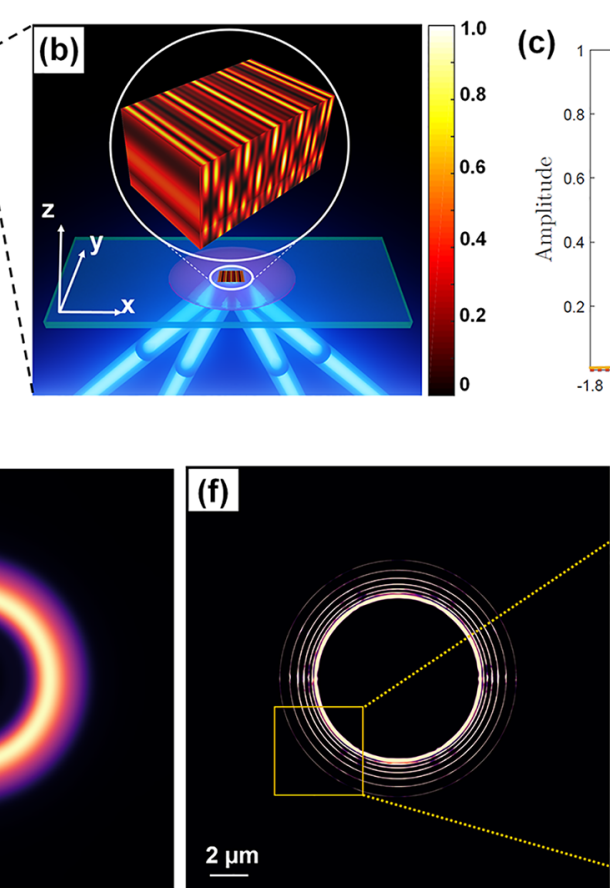

(c)

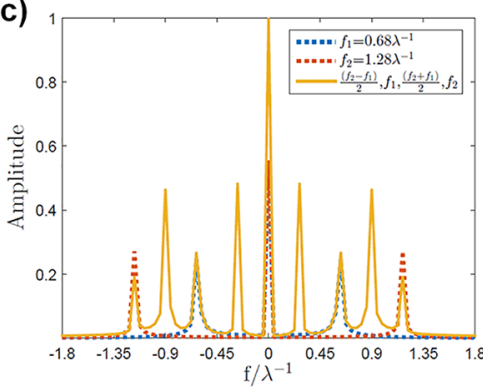

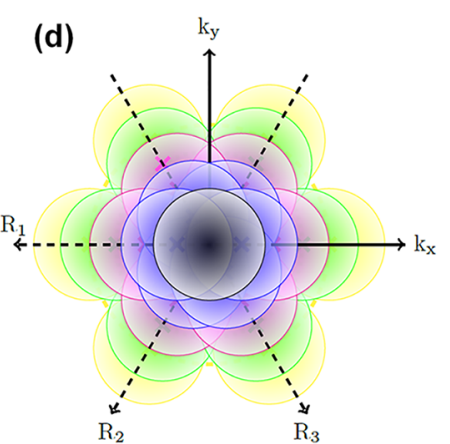
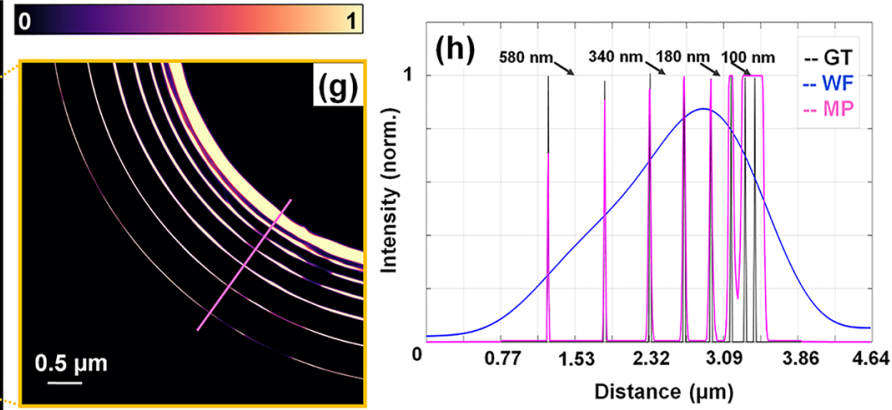

Figure 5. Scheme of multiperiodic lattice illumination and simulation results: (a) graphic of illumination scheme for multiperiodic lattice through interference of four coplanar beams; (b) magnified view of the volumetric interference pattern in the sample (S) region; (c) frequency spectra of the multiperiodic lattice illumination; there is a mixture of four different frequencies $\frac{\left(f_{2}-f_{1}\right)}{2}=0.3 \lambda^{-1}, f_{1}=0.68 \lambda^{-1}, \frac{\left(f_{2}+f_{1}\right)}{2}=0.98 \lambda^{-1}$, and $f_{2}=$ $1.28 \lambda^{-1}$ in the increasing order, respectively; (d) $2 \mathrm{D}$ spectral spread in multiperiodic lattice illumination imaging with isotropic coverage of the higher frequency space in three orientations for numerical aperture NA $=0.3$ and excitation wavelength $=400 \mathrm{~nm}$; (e) diffraction limited widefield image of the sample; (f) MUSICAL reconstructed image for multiperiodic pattern; (g) magnified view of a small portion of (f); (h) radial line-scan of ground-truth, widefield, and MUSICAL images.

lattice that contains more than a single spatial frequency, ${ }^{35}$ simultaneously. The lattice is generated by mutual interference of four coplanar beams where all the four beams are symmetrically arranged around the optic-axis; the wave-vector of the $u$-th beam, $u \in\{-2,-1,+1,+2\}$ in the $R_{j}$-th configuration is given as $\boldsymbol{k}_{u}^{R_{j}}=k\left[\sin \theta_{u} \cos \varphi_{j}, \sin \theta_{u} \sin \varphi_{j}, \cos \right.$ $\left.\theta_{u}\right]$, where $k=\frac{2 \pi}{\lambda_{\mathrm{ex}}}$ is the magnitude of the wave vector, $\theta_{u}$ is the polar angle of the $u$-th beam, and $\varphi_{j}$ is the azimuthal angle of the beams confined in the $R_{j}$-th plane. The mutual interference occurs in a common volumetric region where four coplanar beams overlap with each other. The resultant intensity profile of four linearly polarized beams corresponding to the $R_{j}$ plane is expressed by

$$
I_{j, \delta \nu}(\boldsymbol{r})=\sum_{u=-2}^{+2} \mid \boldsymbol{E}_{u}^{\left.R_{j}\right|^{2}}+\sum_{u} \sum_{\substack{v \\ u \neq v}} \boldsymbol{E}_{u}^{R_{j}} \cdot \boldsymbol{E}_{v}^{R_{j}{ }^{*}} e^{i\left[\left(\boldsymbol{k}_{u}^{R_{j}}-\boldsymbol{k}_{v}^{R_{j}}\right) \cdot \boldsymbol{r}+\left(\psi_{u}-\psi_{v}\right)\right]}
$$

where $\boldsymbol{E}_{u}^{R_{j}}$ is the $u$-th order electric field vector, $\delta \psi=\left(\psi_{u}-\psi_{v}\right)$ is the phase difference of the beams in the $R_{j}$ plane. The graphic of the illumination scheme for the multiperiodic $1 \mathrm{D}$ lattice has been presented in Figure 5a; Figure 5b shows the magnified perspective view of the region of interest around the sample, inset displays volumetric irradiance profile; Figure 5c displays the Fourier spectra of illumination; Figure $5 \mathrm{~d}$ shows isotropy of the illumination in three different planes of interference $R_{1}, R_{2}$, and $R_{3}$ corresponding to three different orientations $\varphi_{1}=0^{\circ}, \varphi_{2}=60^{\circ}$, and $\varphi_{3}=120^{\circ}$, respectively. The first $( \pm 1)$ and second $( \pm 2)$ order beam pairs interfere in the sample plane at angles $\theta_{1}=20^{\circ}$ and $\theta_{2}=40^{\circ}$ w.r.t. the optic axis (i.e., $z$-axis).

The intensity expressions $I_{1, \delta \psi}, I_{2, \delta \psi}$, and $I_{3, \delta \psi}$ represent the illumination data set of multiperiodic lattice for the plane of incidence $R_{1}, R_{2}$, and $R_{3}$, respectively. The phase offset $\psi_{u}$ of the interfering beams in each of the three orientations are modified in such a way (explained in Supporting Information, T4) that it delivers $2 \pi / N$ phase steps to the multiperiodic lattice illumination, where $N$ is the number of phase-shifting considered for the multiperiodic lattice. In the simulation, we have considered $N=10$ acquisitions in each of the three orientations. Each lattice illumination pattern consists of four different spatial frequencies, $\frac{\left(f_{2}-f_{1}\right)}{2}=0.3 \lambda^{-1}, f_{1}=0.68 \lambda^{-1}$, $\frac{\left(f_{2}+f_{1}\right)}{2}=0.98 \lambda^{-1}$, and $f_{2}=1.28 \lambda^{-1}$ in increasing order, respectively, shown in Figure 5c. There are two fundamental frequencies and two sum frequencies obtained from the Fourier transform of the multiperiodic lattice. This type of illumination pattern enables a broad range of spatial frequency space collected by the imaging system. The centered black circle represents the diffraction-limited passband and the four side lobes with blue, pink, green, and yellow color in each of the three orientations correspond to the four different frequency peaks observed in Figure $5 \mathrm{~d}$. This approach contributes toward the enhancement of image resolution due to multiply folding high-frequency information within the passband limit of the microscope. We note a special feature of the multiperiodic lattice: it possesses axial periodicity given by $\frac{\lambda_{\mathrm{ex}}}{\left(\cos \theta_{1}-\cos \theta_{2}\right)}$; which could be useful for light-sheet fluorescence 
microscopy ${ }^{36}$ or three-dimensional imaging. ${ }^{37}$ However, the exploitation of this feature is considered to be beyond the scope of this paper. The objective lens, camera specifications, fluorescence excitation-emission wavelengths, and all other parameters are the same as the ones used for the case of sinusoidal lattice in the previous section. Simulation is performed by taking the same ground-truth sample as in the previous sinusoidal case. The results shown in Figure 5 are for the multiperiodic lattice illumination with MUSICAL reconstruction, where Figure $5 \mathrm{e}$ is the diffraction-limited widefield image of the sample, Figure $5 \mathrm{f}$ is the reconstructed final image using MUSICAL, Figure $5 \mathrm{~g}$ represents the magnified view of a small portion of reconstructed final image, and Figure $5 \mathrm{~h}$ radial line-scan of ground-truth, widefield, and final super-resolved image of the fluorescent sample. From the result, we conclude that even the rings with separation as small as $130 \mathrm{~nm}$ are nicely resolved using multiperiodic lattice illumination.

Table 1 presents a summary of simulation results obtained for one-dimensional lattice illumination microscopy. Tabulated

Table 1. Resolution Obtained Using Various Lattice Illumination for $\lambda_{\mathrm{em}}=450 \mathrm{~nm}$ and $\mathrm{NA}=0.3$ and with and without MUSICAL $^{a}$

\begin{tabular}{llll}
$\begin{array}{c}\text { illumination } \\
\text { scheme }\end{array}$ & \multicolumn{1}{c}{$\begin{array}{c}\text { max. frequency support } \\
\left(\lambda_{\mathrm{em}}^{-1}\right)\end{array}$} & $\begin{array}{c}\text { resolution from } \\
\text { frequency } \\
\text { support only }\end{array}$ & $\begin{array}{c}\text { resolution } \\
\text { from } \\
\text { MUSICAL }\end{array}$ \\
widefield & $2 \mathrm{NA}=0.60$ & $750 \mathrm{~nm}$ (Abbe) & \\
sinusoidal & $2(\mathrm{NA}+\sin \theta)=0.94$ & $478 \mathrm{~nm}$ & $250 \mathrm{~nm}$ \\
multiperiodic & $2\left(\mathrm{NA}+\sin \theta_{2}\right)=1.88$ & $239 \mathrm{~nm}$ & $130 \mathrm{~nm}$
\end{tabular}

${ }^{a_{T}}$ The maximum frequency support is illustrated in the Supporting Information, Figure S8, and the resolution enhancement factor for MUSICAL is explained in the Supporting Information, T7.

data signifies that multiperiodic illumination delivers higher resolution than sinusoidal illumination, as it simultaneously folds higher spatial frequencies within the optical passband limit. The maximum frequency support for sinusoidal and multiperiodic lattice illumination is $0.94 \lambda_{\mathrm{em}}^{-1}(1.5 \times$ widefield $)$ and $1.88 \lambda_{\mathrm{em}}^{-1}$ ( $3 \times$ widefield), respectively. This blind reconstruction approach extends the frequency support of the final image which eventually leads to 3- and 6-fold resolution enhancement over diffraction limit in sinusoidal and multiperiodic illumination, respectively. A further mathematical analysis of frequency support of MUSICAL is provided in the Supporting Information, T7 for the interested readers.

\section{CONCLUSION}

Thus, in summary, a super-resolution optical lattice illumination microscopy is presented with both sinusoidal and multiperiodic lattice illumination patterns, generated by the interference of coplanar beams. The advantage of using MUSICAL in close conjugation with such lattice illumination is explained alongwith in-depth discussions on this particular algorithm of interest. The sinusoidal and multiperiodic illumination pattern along with MUSICAL reconstruction delivers maximum 3- and 6-fold resolution enhancement over the diffraction limit, respectively. Such a demonstration opens up the chances to use different types of periodic illumination toward achieving super-resolution through the use of MUSICAL-like reconstruction algorithm. However, for such considerations, the only criteria required to be satisfied is the uniformity of the average and the standard deviation of several raw frames across the sample plane. Apart from theoretical considerations, a demonstration of MUSICAL on experimentally recorded fluorescent beads with sinusoidal lattice successfully confirms the super-resolution imaging abilities in lesser number of processing steps, further validated over different experimental data sets. Additionally, the multiperiodic illumination pattern generated by the interference of four coplanar beams containing both lateral $(x-y)$ as well as axial $(z)$ periodicity offer potential new approaches in threedimensional super-resolution imaging and light-sheet microscopy for future explorations.

\section{ASSOCIATED CONTENT}

\section{Supporting Information}

The Supporting Information is available free of charge at https://pubs.acs.org/doi/10.1021/acsphotonics.1c00503.

Summary of the MUSICAL algorithm, complete set of eigen-images and eigen-spectra, the performance of MUSICAL for the lattice illumination, scheme of the phase-modulated multiperiodic lattice illumination, the experimental work, analysis of tubulin data, frequency support of MUSICAL (PDF)

\section{AUTHOR INFORMATION \\ Corresponding Authors \\ Joby Joseph - Department of Physics, Indian Institute of Technology Delhi, New Delhi 110016, India; (1) orcid.org/ 0000-0002-1513-8008; Email: joby@physics.iitd.ac.in \\ Krishna Agarwal - Department of Physics and Technology, UiT-The Arctic University of Norway, Tromsø 9037, Norway; Email: krishna.agarwal@uit.no}

\section{Authors}

Krishnendu Samanta - Department of Physics, Indian Institute of Technology Delhi, New Delhi 110016, India

Swagato Sarkar - Department of Physics, Indian Institute of Technology Delhi, New Delhi 110016, India; Leibniz-Institut fü Polymerforschung Dresden e.V. (IPF), Institute for Physical Chemistry and Polymer Physics, 01069 Dresden, Germany; $\odot$ orcid.org/0000-0002-6979-3446

Sebastian Acuña - Department of Physics and Technology, UiT-The Arctic University of Norway, Tromsø 9037, Norway

Balpreet Singh Ahluwalia - Department of Physics and Technology, UiT-The Arctic University of Norway, Tromsø 9037, Norway; ㅇo이.org/0000-0001-7841-6952

Complete contact information is available at:

https://pubs.acs.org/10.1021/acsphotonics.1c00503

\section{Author Contributions}

K.S. and S.S. have constructed the manuscript and the necessary figures. K.S. has performed all the simulations. S.A. carried out the eigenimage analysis. B.S.A., J.J., and K.A. supervised the whole work. All the authors provided critical feedback and helped to shape the research, analysis, and manuscript.

\section{Funding}

This work is supported by the Indo Norwegian Collaborative Program (Project Number - INCP 2014/10024), University Grants Commission (UGC, India), Department of Science and Technology (DST, India), Freigeist project (Germany), Horizon 2020 Marie Skłstrokodowska-Curie Action (SEP- 
210382872), Horizon 2020 ERC Starting Grant (804233), Research Council of Norway FRIPRO Young Project (288082).

\section{Notes}

The authors declare no competing financial interest.

\section{ACKNOWLEDGMENTS}

K.S. acknowledges the University Grants Commission (UGC, India), S.S. acknowledges the Department of Science and Technology, Govt. of India for financial aiding through the INSPIRE Fellowship, and Dr. Habil. Tobias A.F. König for support through a salary from Freigeist Fellowship, Volkswagen Foundation, Germany; K.A. acknowledges funding from a Horizon 2020 Marie Skłodowska-Curie Actions (SEP210382872), a Horizon 2020 ERC Starting Grant (804233), and a Research Council of Norway FRIPRO Young Project (288082).

\section{REFERENCES}

(1) Abbe, E. XV.-The Relation of Aperture and Power in the Microscope (continued). J. R. Microsc. Soc. 1883, 3, 790-812.

(2) Zheludev, N. I. What diffraction limit? Nat. Mater. 2008, 7, 420422.

(3) Hell, S. W. Far-field optical nanoscopy. Science 2007, 316, 11531158.

(4) Nieuwenhuizen, R. P.; Lidke, K. A.; Bates, M.; Puig, D. L.; Grünwald, D.; Stallinga, S.; Rieger, B. Measuring image resolution in optical nanoscopy. Nat. Methods 2013, 10, 557-562.

(5) Manley, S.; Gillette, J. M; Patterson, G. H; Shroff, H.; Hess, H. F; Betzig, E.; Lippincott-Schwartz, J. High-density mapping of singlemolecule trajectories with photoactivated localization microscopy. Nat. Methods 2008, 5, 155-157.

(6) Arnspang, E. C.; Sengupta, P.; Mortensen, K. I.; Jensen, H. H.; Hahn, U.; Jensen, E. B. V.; Lippincott-Schwartz, J.; Nejsum, L. N. Regulation of Plasma Membrane Nanodomains of the Water Channel Aquaporin-3 Revealed by Fixed and Live Photoactivated Localization Microscopy. Nano Lett. 2019, 19, 699-707.

(7) Rust, M. J.; Bates, M.; Zhuang, X. Sub-diffraction-limit imaging by stochastic optical reconstruction microscopy (STORM). Nat. Methods 2006, 3, 793-796.

(8) Xu, J.; Tehrani, K. F.; Kner, P. Multicolor 3D super-resolution imaging by quantum dot stochastic optical reconstruction microscopy. ACS Nano 2015, 9, 2917-2925.

(9) Blom, H.; Widengren, J. Stimulated Emission Depletion Microscopy. Chem. Rev. (Washington, DC, U. S.) 2017, 117, 73777427.

(10) Sivan, Y.; Sonnefraud, Y.; Kena-Cohen, S.; Pendry, J. B.; Maier, S. A. Nanoparticle-assisted stimulated-emission-depletion nanoscopy. ACS Nano 2012, 6, 5291-5296.

(11) Fölling, J.; Bossi, M.; Bock, H.; Medda, R.; Wurm, C. A.; Hein, B.; Jakobs, S.; Eggeling, C.; Hell, S. W. Fluorescence nanoscopy by ground-state depletion and single-molecule return. Nat. Methods 2008, 5, 943-945.

(12) Han, K. Y.; Kim, S. K.; Eggeling, C.; Hell, S. W. Metastable dark states enable ground state depletion microscopy of nitrogen vacancy centers in diamond with diffraction-unlimited resolution. Nano Lett. 2010, 10, 3199-3203.

(13) Heintzmann, R.; Huser, T. Super-resolution structured illumination microscopy. Chem. Rev. (Washington, DC, U. S.) 2017, 117, 13890-13908.

(14) Gustafsson, M. G. L. Surpassing the lateral resolution limit by a factor of two using structured illumination microscopy. J. Microsc. (Oxford, U. K.) 2000, 198, 82-87.

(15) Chen, K. Y.; Lee, A. T.; Hung, C. C.; Huang, J. S.; Yang, Y. T. Transport and Trapping in Two-Dimensional Nanoscale Plasmonic Optical Lattice. Nano Lett. 2013, 13, 4118-4122.
(16) Grynberg, G.; Lounis, B.; Verkerk, P.; Courtois, J. Y.; Salomon, C. Quantized motion of cold cesium atoms in two- and threedimensional optical potentials. Phys. Rev. Lett. 1993, 70, 2249-2252.

(17) Dumke, R.; Volk, M.; Müther, T.; Buchkremer, F. B. J.; Birkl, G.; Ertmer, W. Micro-optical Realization of Arrays of Selectively Addressable Dipole Traps: A Scalable Configuration for Quantum Computation with Atomic Qubits. Phys. Rev. Lett. 2002, 89, 097903.

(18) Betzig, E. Excitation strategies for optical lattice microscopy. Opt. Express 2005, 13, 3021-3036.

(19) Müller, M.; Mönkemöller, V.; Hennig, S.; Hübner, W.; Huser, $\mathrm{T}$. Open-source image reconstruction of super-resolution structured illumination microscopy data in ImageJ. Nat. Commun. 2016, 7, 10980.

(20) Lal, A.; Shan, C.; Xi, P. Structured Illumination Microscopy Image Reconstruction Algorithm. IEEE J. Sel. Top. Quantum Electron. 2016, 22, 50-63.

(21) Yeh, L. H.; Tian, L.; Waller, L. Structured illumination microscopy with unknown patterns and a statistical prior. Biomed. Opt. Express 2017, 8, 695-711.

(22) Lahrberg, M.; Singh, M.; Khare, K.; Ahluwalia, B. S. Accurate estimation of the illumination pattern's orientation and wavelength in sinusoidal structured illumination microscopy. Appl. Opt. 2018, 57, $1019-1025$.

(23) Agarwal, K.; Machan, R. Multiple signal classification algorithm for super-resolution fluorescence microscopy. Nat. Commun. 2016, 7, 13752.

(24) Sekh, A. A.; Opstad, I. S.; Birgisdottir, A. B.; Myrmel, T.; Ahluwalia, B. S.; Agarwal, K.; Prasad, D. K. Learning Nanoscale Motion Patterns of Vesicles in Living Cells. Proc. IEEE Comput. Soc. Conf. Comput. Vis. Pattern Recognit 2020, 14011-14023.

(25) Min, J.; Jang, J.; Keum, D.; Ryu, S.-W.; Choi, C.; Jeong, K.-H.; Ye, J. C. Fluorescent microscopy beyond diffraction limits using speckle illumination and joint support recovery. Sci. Rep. 2013, 3, 2075.

(26) Mudry, E.; Belkebir, K.; Girard, J.; Savatier, J.; Le Moal, E.; Nicoletti, C.; Allain, M.; Sentenac, A. Structured illumination microscopy using unknown speckle patterns. Nat. Photonics 2012, 6, $312-315$.

(27) Lochocki, B.; Abrashitova, K.; de Boer, J. F.; Amitonova, L. V. Ultimate resolution limits of speckle-based compressive imaging. Opt. Express 2021, 29, 3943-3955.

(28) Jayakumar, N.; Helle, Ø. I.; Agarwal, K.; Ahluwalia, B. S. Onchip TIRF nanoscopy by applying Haar wavelet kernel analysis on intensity fluctuations induced by chip illumination. Opt. Express 2020, $28,35454-35468$.

(29) Zhao, G.; Zheng, C.; Kuang, C.; Liu, X. Resolution-enhanced SOFI via structured illumination. Opt. Lett. 2017, 42, 3956-3959.

(30) Sarkar, S.; Samanta, K.; Joseph, J. Method for single-shot fabrication of chiral woodpile photonic structures using phasecontrolled interference lithography. Opt. Express 2020, 28, 43474361.

(31) Dertinger, T.; Colyer, R.; Vogel, R.; Enderlein, J.; Weiss, S. Achieving increased resolution and more pixels with Superresolution Optical Fluctuation Imaging (SOFI). Opt. Express 2010, 18, 1887518885 .

(32) Acuña, S.; Ströhl, F.; Opstad, I.; Ahluwalia, B. S.; Agarwal, K. MusiJ: an ImageJ plugin for video nanoscopy. Biomed. Opt. Express 2020, 11, 2548-2559.

(33) Demmerle, J.; Innocent, C.; North, A. J.; Ball, G.; Müller, M.; Miron, E.; Matsuda, A.; Dobbie, I. M.; Markaki, Y.; Schermelleh, L. Strategic and practical guidelines for successful structured illumination microscopy. Nat. Protoc. 2017, 12, 988-1010.

(34) Jadhav, S.; Acuña, S.; Opstad, I. S.; Ahluwalia, B. S.; Agarwal, K.; Prasad, D. K. Artefact removal in ground truth deficient fluctuations-based nanoscopy images using deep learning. Biomed. Opt. Express 2021, 12, 191-210.

(35) Shterman, D.; Gjonaj, B.; Bartal, G. Experimental demonstration of multi moiré structured illumination microscopy. ACS Photonics 2018, 5, 1898-1902. 
(36) Vettenburg, T.; Corral, A.; Rodriguez-Pulido, A.; Flors, C.; Ripoll, J. Photoswitching-Enabled Contrast Enhancement in Light Sheet Fluorescence Microscopy. ACS Photonics 2017, 4, 424-428.

(37) Schermelleh, L.; Carlton, P. M.; Haase, S.; Shao, L.; Winoto, L.; Kner, P.; Burke, B.; Cardoso, M. C.; Agard, D. A.; Gustafsson, M. G. L.; Leonhardt, H.; Sedat, J. W. Subdiffraction Multicolor Imaging of the Nuclear Periphery with 3D Structured Illumination Microscopy. Science 2008, 320, 1332-1336.

(38) Acuña, S.; Roy, M.; Villegas-Hernandez, L.; Dubey, V. K.; Ahluwalia, B. S.; Agarwal, K. Deriving high contrast fluorescence microscopy images through low contrast noisy image stacks. Biomed. Opt. Express 2021, na. 